\title{
Design Features of a Precision AC-DC Converter
}

\author{
Louis A. Marzetta \\ Institute for Applied Technology, National Bureau of Standards, Washington, D.C. 20234 \\ and \\ Donald R. Flach \\ Institute for Basic Standards, National Bureau of Standards, Washington, D.C. 20234 \\ (August 5, 1969)

\begin{abstract}
With the availability today of high performance operational amplifiers and related components, it is possible to construct an instrument for ac-de transfer work that meets the requirements of a standards laboratory. Transformation of measured average a-c values into d-c voltage is possible with a predictable accuracy of 20 parts per million up to $1 \mathrm{kHz}$. Precision of operation is assured from dc to $100 \mathrm{kHz}$.
\end{abstract}

Key words: AC; DC; operational; precision; rectifier; transfer.

\section{Introduction}

Reference to precise a-c measurement has always implied an rms evaluation. This has been so because the majority of physical results in experiments and other usage are related to transformation of electrical energy, to heat or mechanical work, and electrical laboratories have found the best accuracy in squarelaw responding instruments. With the availability today of highly stable, nearly pure sine-wave power sources and precision linear operational devices, the situation has changed. For some ac-dc transfer work such as amplitude monitoring [1], ${ }^{1}$ the average a-c responding systems to be described can have a superiority of precision over rms converters. In addition, the wide usage of average responding instruments such as digital voltmeters with a-c options and sampling devices has increased the need for a basic average transfer standard. This paper will delve into design factors that demonstrate the ability of transforming the average value into $d$-c voltage with a high order of accuracy.

\section{Operational Rectifier Stage}

The choice of systems and circuits for average a-c measurement is more limited than for rms. An average

${ }^{1}$ Figures in brackets indicate the literature references at the end of this paper. responding circuit is involved with some form of rectification - that is, it has to average either the positive or the negative values of the voltage wave, or both without regard to sign. It is the defects in the rectifying elements that affect precision and accuracy. The object of the operational rectifier circuit, figure 1 , is to place the rectifying components in the feedback loop at the output of the amplifier wherein the full loop gain amplification can be used to greatly reduce the diode weaknesses. The device is a unity gain circuit (if equal value resistors are used at the input and feedback connections) whereby half-sine waves are generated at each output.

Because of the unwieldy nature of transfer expressions in circuits with diodes, a linear amplifier type analysis in the frequency domain will be pursued first, by neglecting the diode imperfections.

\subsection{Finite Gain Error}

Using superposition theory and writing a current balance at the input summing junction, we find that the transfer function of a unity-gain inverting operational amplifier with perfect ratio resistors is

$$
V_{\text {out }} / V_{\text {in }}=-\frac{1}{1+\left(2 / A_{f}\right)}
$$

where $A_{f}$ is the open-loop voltage amplification of an 


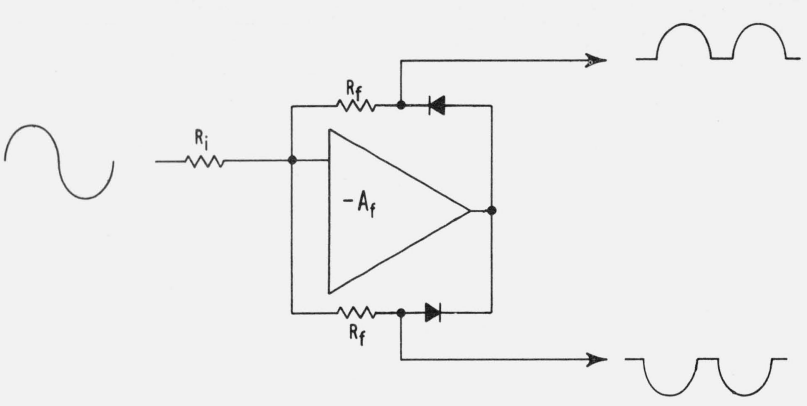

FIGURE 1. Basic operational rectifier.

operational amplifier with ideal characteristics but finite amplification.

To avoid commonly observed errors in the usual exact-gain formula due to neglect of the phasor nature of the open-loop voltage amplification, $A_{f}$, the general formula can be expressed in a more usable form as follows

$$
\begin{aligned}
V_{\text {out }} / V_{\text {in }} & =-\left(Z_{f} / Z_{i}\right) \\
& \cdot \frac{1}{1+\left(1 / A_{f}\right)(\cos \theta-j \sin \theta)\left[1+\left(Z_{f} / Z_{i}\right)\right]}
\end{aligned}
$$

where $\theta$ is the open loop phase angle of the amplifier, and $A_{f}$ is now the magnitude of the open loop amplification. Starting with zero degrees at direct current, $\theta$ usually increases with a minus sign (lag) as the frequency increases. $Z_{f}$ and $Z_{i}$ are feedback and input impedances, respectively. In most literature a reader tends to interpret the closed-loop gain expression as an output that falls off with frequency because of the drop in open loop amplification. Careful tests corroborate expression (2) and show that this is not necessarily so. The output can actually rise with frequency for a constant input in spite of a dropping $A_{f}$ in the region where $\theta$ has shifted beyond 90 degrees lag, the reason being that the phase lag has created a real component of positive feedback.

The transfer error in ppm (parts per million) for an inverting unity gain situation is the difference between unity and the magnitude of $V_{\text {out }} / V_{\text {in }}$. Except for angles near $90^{\circ}$, the error can be closely approximated as $\pm\left(2 / A_{f}\right) \cdot(\cos \theta) \cdot 10^{6}$; the minus sign for phase lag less than $90^{\circ}$ and plus sign for phase lag greater than $90^{\circ}$, where $\left(A_{f} \gg 1\right)$. Exactly at $90^{\circ}$ the error reduces to $2 / A_{f}^{2}$, and reaches zero error slightly beyond $90^{\circ}$. However, effectiveness of quadrature error reduction does drop off rapidly for phase deviations from the $90^{\circ}$ region.

Since phase characteristics are rarely given in operational amplifier literature, the transfer error calculation of the operational rectifier will be hampered. Using the worst case basis of $\left(2 / A_{f}\right) \cdot 10^{6}$ for the error, for an amplifier with zero phase shift, it could be expected that an operational amplifier would exhibit a gain transfer error of $100 \mathrm{ppm}$ at the frequency where the open loop amplification is 20000 and that the error would double each octave increase of frequency. However, it was found from actual test data that the error function did not behave in this manner but that the output remains quite constant for a wide frequency range, in some cases rising at the higher frequencies due to the previously described phase properties. Hence it can be assumed that the $\left(2 / A_{f}\right) \cdot 10^{6}$ error factor is quite conservative.

\subsection{Finite Diode Conduction Level Error}

Another source of error arises from the finite conduction level of the diodes. Time is lost at the amplifier in order for its output to reach the diode conduction voltage, which means that there is a "lost area" at the beginning of the half sine wave output. The overall error in average value with a first order approximation can be expressed as the ratio of the lost small area to the half-wave area. It is desirable to analyze the factors that may influence this particular error.

For the period of a half-sine wave with $V_{m}$ peak volts, the average value is $(2 / \pi) V_{m}$. Hence the area under the function is $(2 / \pi)\left(V_{m}\right)(\pi)$ or $2 V_{m}$ volt-radians. The lost area in question can be found by formal integration as

$$
\text { Lost Area }=V_{m} \int_{0}^{\phi_{c}} \sin \phi d \phi
$$

where $\phi_{c}$ is the angle measured from the zero crossing point to the diode conduction point. For small angles, $\cos \phi=1-\left(\phi^{2} / 2\right)$ and the lost area from expression (4) is

Lost Area $=V_{m}|-\cos \phi|{ }_{0}^{\phi_{c}}=V_{m}\left(\phi_{c}^{2} / 2\right)$ volt-radians.

At angle $\phi_{c}$ the diode conduction voltage, $V_{c}$, at the amplifier output is

$$
V_{c}=A_{f} V_{s}
$$

where $V_{s}$ is the potential at the operational amplifier's summing input junction and $A_{f}$ is the open-loop voltage amplification. Also for small radian angles

$$
V_{s}=\phi_{c} V_{m}
$$

Substituting (7) into (6), the angle at conduction point becomes

$$
\phi_{c}=V_{c} / V_{m} A_{f} .
$$

Using expressions (5) and (8)

$$
\text { Lost Area }=V_{c}^{2} / 2 V_{m} A^{2} \text {. }
$$

Taking the ratio of the lost area (9) to the half-wave area $\left(2 V_{m}\right)$, the overall error is

$$
\text { error, } \epsilon=V_{c}^{2} / 4 V_{m}^{2} A_{j}^{2} \text {. }
$$

Because of the squared value for $A_{f}$ in the expression, 
the error due to the finite diode conduction level is considerably less than the closed-loop gain error noted in expression (2).

\subsection{Slew Rate Error}

A second source of possible error from the diode conduction level is found in the consideration of the operational amplifier-rectifier in the time domain. Included in the data on operational amplifiers is the "slew rate"-a measure of the device's transient response. Slewing rate, $S$, or maximum output voltage change with respect to time is expressed as

$$
S=\left(d V_{0} / d t\right)_{\max }=V_{p}\left(\omega_{h} / 10^{6}\right) \text { volts per microsecond }
$$

where $V_{p}$ is the peak output voltage and $\omega_{h}$ is the maximum frequency in radians per second for full output of the device. Using the stated slew rate, the time lost in reaching diode conduction can be found and used to compute the lost area. The elapsed time $\left(t_{c}\right)$ from zero crossing to conduction, $V_{c}$ is

$$
t_{c}=V_{c} / S \text { seconds. }
$$

The angle, $\phi_{c}$, of expression (4) can be expressed as

$$
\phi_{c}=\left(t_{c} / t_{0}\right)(2 \pi) \text { radians }
$$

where $t_{0}$ is the time for one period of the operating frequency, $f_{0}$. Substituting (12) in (13)

$$
\phi_{c}=V_{c} 2 \pi f_{0} / S \text { radians }
$$

Using the lost area expression (5) and expression (14) for $\phi_{c}$

$$
\text { Lost area due to } S=V_{m}\left[V_{c} 2 \pi f_{0} / S\right]^{2} / 2 \text {. }
$$

As in expression (10) the overall error, $\epsilon$, comes from the ratio of lost area to half-wave area.

$$
\text { error, } \boldsymbol{\epsilon}=V_{c}^{2}\left(2 \pi f_{0}\right)^{2} / S^{2} \text {. }
$$

Comparing expressions (16) and (10)-for example: if $f_{0}=16 \mathrm{kHz}, V_{c}$ is about $0.6 \mathrm{~V}$, an amplifier with $A_{f}=10^{4}$ and a slew rate of $10 \mathrm{~V}$ per microsecond, the error from expression (10) with an input signal of $V_{m}=7 \mathrm{~V}$ becomes approximately two parts in $10^{11}$. Using expression (16) the error is about ten parts in $10^{6}$.

In this example the slew rate factor contributed a greater error than the $A_{f}$ gain factor. However, the slew rate figure alone is not a reliable measure of an amplifier's ability to be used as a precision operational rectifier. It is possible for a device to have both a relatively low $A_{f}$ and a high slew rate since the two are independent design features of the amplifier. Such a rectifier would suffer a possible error from expression (10). Fortunately all amplifiers investigated that have the desired open-loop ampli- fication also have sufficient slew rate. One additional note is that amplifier output overshoot at the beginning and end of the half-sine wave has not been a troublesome factor.

Thus far the discussion of the operational rectifier has been concerned with its a-c requirements; however the precision of its average value output also demands a strict de stability. Not very long ago it was possible to achieve low drift results only with chopper-stabilized devices. High a-c performance operational amplifiers are now available in differential, nonchopper design wherein the drift error contribution with a $5 \mathrm{~V}$ input signal can be kept below $20 \mathrm{ppm}$ for hours. Bias current variations at the input are low enough so as to be negligible in producing $d$-c output variations with feedback resistors encountered ( $1 \mathrm{k} \Omega$ to $100 \mathrm{k} \Omega$ ).

\subsection{Feed-Forward Operational Amplifiers}

In the course of experimenting with a number of available operational amplifiers a certain group of them were found to exhibit a marked anomalous behavior. These particular amplifiers showed a monotonically decreasing response with frequency that was much sharper than was predictable with the conservative error factor bases. An inquiry into design of these amplifiers revealed them as "feedforward" type devices. Briefly this means the input signal assumes different paths through the overall amplifier chain depending upon the input frequency, in the manner of a chopper stabilized amplifier. They can sometimes be spotted in the literature as wide-band devices with a high capacitance at the inverting input, as much as $0.02 \mu \mathrm{F}$. While feedforward type operational amplifiers can be used as operational rectifiers, FET differential amplifiers characterized by their low input capacitance at both inputs ( $5 \mathrm{pF}$ or less) are generally preferred.

\section{Operational Filter Stage}

The operational stage following the rectifier is the filter. (Fig. 2) Basically it is an accurate unity gain stage with a large enough time constant to filter out the half-sine-wave ripple to a level tolerable to the d-c detector. It also provides nearly ideal impedance buffering between the rectifier output and the d-c detector. The filter operational amplifier a-c response performance is unimportant, but like the operational rectifier, the need for low d-c voltage drift must be met. To preserve the unity gain accuracy and to present a low output impedance, the open loop d-c voltage amplification should be at least $200000-$ a figure that is easily found in today's devices.

The feedback filter capacitor should have enough capacitance to achieve the desired time constant in conjunction with the feedback resistor ( $10 \mathrm{k} \Omega$ to $50 \mathrm{k} \Omega$ ) to meet the detector requirements. Capacitor leakage resistance is important; the value should be at least $10^{5}$ times the feedback resistor value. Polycarbonate dielectric capacitors are available in a $40 \mu \mathrm{F}$ value, meeting the low leakage requirements. 


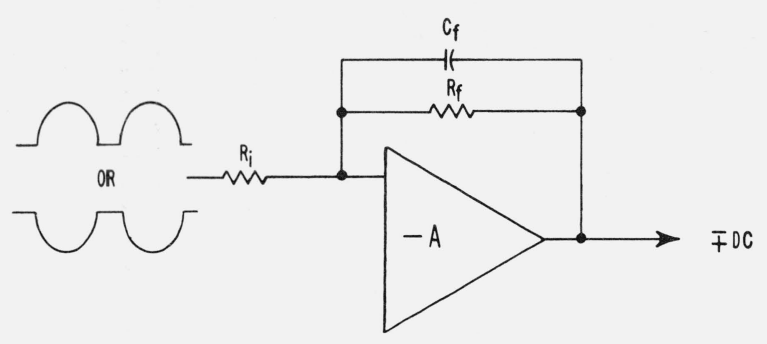

FiguRe 2. Basic operational filter.

\section{Choice of Precision Resistors for the Rectifier and Filter Stages}

The principal factor in the gain-setting accuracy of an operational stage is the ratio accuracy of the circuit resistors external to the component amplifier. Wirewound resistors of various forms are available with both individual and ratio accuracies of $5 \mathrm{ppm}$. Extensive tests have demonstrated their ability to perform well in operational circuits. With little complexity it is possible to detect by $\mathrm{d}$-c null techniques a difference of less than $5 \mathrm{ppm}$ in a pair of high quality wire wound resistors by interchanging their respective positions at the input and feedback of an operational amplifier. The problem with some wire wound resistors is that they can exhibit a noticeable dc-ac error for frequencies above $5 \mathrm{kHz}$, amounting to several hundred ppm at $50 \mathrm{kHz}$. While it is possible to partially compensate for the error by using networks tailored to the resistor characteristics, it is the purpose of this article to describe systems of high precision that are void of compensation schemes. Fortunately there are available metal film resistors with desirable properties for the immediate application. These possess a virtually zero dc-ac difference over the range of interest ( $\mathrm{dc}$ to $100 \mathrm{kHz}$ ). They are readily available with better than $100 \mathrm{ppm}$ ratio accuracy with a relative temperature coefficient of a few ppm per degree. For wide band work in the area of $100 \mathrm{ppm}$ accuracy, the metal film resistors are highly recommended; for high-accuracy, low-frequency applications of say 20 ppm, wire wound resistors may be more suitable.

\section{Selection of Diodes at Operational Rectifier}

The role of reverse leakage current and junction capacitance of a chosen diode must be considered in relation to the feedback resistor. It is possible to use a single silicon diode in each feedback path of the rectifier circuit since they are available with leakage currents less than $10^{-9} \mathrm{~A}$. The usual operational forward diode current is $10^{-3} \mathrm{~A}$, hence the error is $1 \mathrm{ppm}$ for the stated leakage current. A more conservative approach is to use a pair of diodes in series, with a resistive shunt path between them for the leakage current; an arrangement conceived by Richman [1]. The series feature also reduces the resultant capacitance at full reverse bias; typically $1 \mathrm{pF}$.
The junction capacitance at the diode can introduce an error by adding a component of reverse current to the diode's forward signal current. However the net effect can be small because of the quadrature phase relationship of the currents. The error expression can be derived from the circuit impedances in the current branches. For small values of capacitance or high ratio of capacitive reactance to series feedback resistance, the error becomes $=1 / 2\left(R_{f} / X_{c}\right)^{2} \cdot 10^{6} \mathrm{ppm}$. For example at an operating frequency of $16 \mathrm{kHz}$ and a feedback resistance of $10^{4} \Omega$, a $1 \mathrm{pF}$ diode capacitance would introduce an error of about $1 / 2 \mathrm{ppm}$.

With two silicon diodes in series the output must reach about $1.2 \mathrm{~V}$ before conduction takes place. This quadruples the small error in average value because of the "lost area" as explained previously. The latest development of "hot carrier" diodes can be used to good advantage. These high-speed diodes have the low-leakage feature of silicon diodes and a low forward conduction voltage similar to germanium less than $0.39 \mathrm{~V}$.

Reverse recovery or effective minority carrier lifetime in a diode is a measure of its ability to turn off in going from forward to reverse conduction. In the operational rectifier this factor affects the termination of the half sine wave function, resulting in an error in average value. The analysis of the error is nearly the same as for the case of limited amplifier slew rate discussed previously with the error showing up as a lost area. Fortunately the diodes described in this section have recovery times in the vicinity of $10^{-9} \mathrm{~s}$. Using the same procedure as with the example of the slew rate discussion, the error contributed at $16 \mathrm{kHz}$ with the stated recovery time is found from expression (6). The error is equal to $\theta^{2} / 4$ or $\left(10^{5} \cdot 10^{-9}\right)^{2} / 4=10^{-8} / 4$ which is negligible.

\section{Stabilization of Operational Rectifier}

To prevent high frequency oscillation a small capacitor is generally needed across the feedback resistor of an operational device. The resultant time constant places an upper corner frequency, $F_{h}$, having a direct bearing on the gain accuracy at some lower operating frequency, $F_{0}$. For large separation of the two frequencies, the asymptotic slope error is nearly $\left(F_{0} / F_{h}\right)^{2} \cdot 10^{6}$, in ppm where $F_{h}=1 / 2 \pi R_{f} C_{f}$. The analysis is correct for a linear system; however in the rectifier configuration the capacitor is across both the feedback resistor and diode string resulting in a marked reduction of corner frequency, $F_{h}$ which is difficult to calculate. Experience has shown that a series pair of $5 \mathrm{pF}$ capacitors has been effective in stabilizing all amplifiers tested. However, this value of stabilizing capacitance can degrade the gain accuracy by $50 \mathrm{ppm}$ at $20 \mathrm{kHz}$ with a $5 \mathrm{k} \Omega$ feedback resistance.

\section{Unity-Gain Input Isolation Amplifier Stage}

The combination of the operational rectifier stage and filter stage is sufficient to meet the needs of most 
average ac-dc transfer requirements. This system has an input impedance equal to the input resistor of the operational rectifier stage, typically $5 \mathrm{k} \Omega$. In some cases this may be annoying because of the loading affect on a-c sources and a-c attenuators. For these reasons the effectiveness of an operational unity-gain impedance transforming circuit was investigated for use in applications where $100 \mathrm{ppm}$ accuracy is sufficient.

The basic circuit is shown in figure 3 . It is recognized as a noninverting amplifier with unity gain for the indicated input-output connection. Note that no precision resistors are involved in its operation and that it is able to take advantage of the high input impedance available with FET type differential devices-around $10^{11} \Omega$ shunted by $3.5 \mathrm{pF}$ of capacitance. In order for the stage to have the desired gain accuracy from $d$-c through the highest operating frequency, the selected operational differential amplifier must have both a wide-band high open loop amplification and a wide-band high common-mode rejection ratio, CMRR. The latter factor is a measure of the amplifier's ability to precisely respond only to the instantaneous amplitude difference value between the two input terminals; hence it desirably produces no output when both inputs are at the same potential and polarity. Analytically the CMRR of a differential amplifier is the ratio of a voltage $V_{m}$ to the difference of the potentials at the two inputs required for a null output; expressed as

$$
\mathrm{CMRR}=V_{n} /\left[\left(V_{n} \pm e_{+}\right)-\left(V_{n} \pm e_{-}\right)\right] .
$$

Each of the terms in the denominator of (17) is an equivalent potential at each input consisting of a magnitude $V_{n}$ and an apparent error potential, $\pm e$. For example if $V_{n}$ is $1 \mathrm{~V}$ and the difference of the two equivalent input potentials is $0.001 \mathrm{~V}$, the CMRR is $1000: 1$. Its polarity is a function of the polarity of the symmetry inperfections, $e_{+}$and $e_{-}$. Writing circuit voltage loops, the transfer function of the stage is expressed as

$$
V_{\text {out }} / V_{\text {in }}=\frac{1 \pm 1 / \text { CMRR }}{1+(\cos \theta-\ddot{j} \sin \theta) / A_{f}}
$$

The effect of wide band CMRR and $A_{f}$ with phase lag that is greater than $90^{\circ}$, can be seen in figure 4 for three situations: (A) high CMRR and low $A_{f}$; (B) high $A_{f}$ and low CMRR; (C) both CMRR and $A_{f}$ are high. The figure is a plot from laboratory data on actual devices and corroborates and predicted behavior

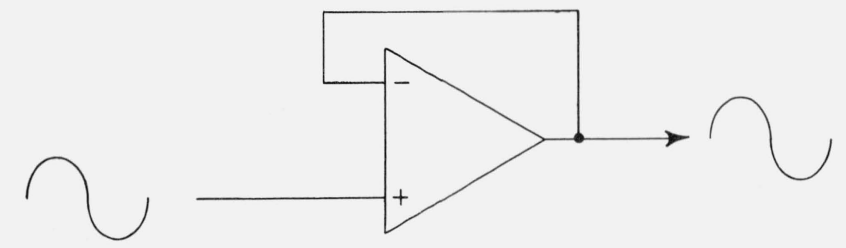

FigURE 3. Unity-gain isolation amplifier. from expression (18) though the slope of curve (B) could have been lower for a minus polarity effect of the CMRR. The three devices shown in figure 4 are high-performance units, and the use of the terms "low $A_{f}$ " or "low CMRR are in a relative sense, keeping in mind that the total ordinate value is less than 0.1 percent error.

The stage also has a desirable low output impedance-a dynamic impedance equal to the amplifier intrinsic output impedance divided by the value of $A_{f}$. A typical amplifier is capable of delivering \pm 10 $V$ output at $10^{6} \mathrm{~Hz}$, with a load current of $10 \mathrm{~mA}$. In summary, the operational stage described is a rather elegant impedance transformer with an impedance ratio of at least $10^{6}: 1$ operating from de through $100 \mathrm{kHz}$ with a gain accuracy of $200 \mathrm{ppm}$ to at least $20 \mathrm{kHz}$.

\section{Test Results}

A large number of tests were performed involving a variety of operational amplifiers for use as precision rectifier, filter, and unity-gain impedance coupling stages. The tests could be grouped in three broad areas of interest: accuracy at a reference frequency, precision (stability and repeatability) and frequency response (ac-dc deviation).

\subsection{Tests for Accuracy}

The first group of tests were concerned with the accuracy of transformation of ac to de by an operational rectifier; i.e., the relation between the average value of the output (half-wave) and the rms value of the input, with a voltage wave of very low distortion, so that $V_{\text {ave }}=V_{\text {rms }} \cdot \sqrt{2} / \pi$. The operational amplifiers available for these tests were of the chopperstabilized type having very high open-loop amplification at frequencies below $5 \mathrm{kHz}$. Their d-c drift was less than $5 \mu \mathrm{v}$ for periods of several hours. Oilfilled, wire-wound resistors matched to a few ppm in unity-ratio value were employed, with resistance values from 1 to $25 \mathrm{k} \Omega$. The major components of the measuring system for these tests, shown in figure 5, included a low-distortion, highly stable a-c power source, (total harmonic distortion below 0.01\%) a seven-dial calibrated, precision d-c power supply, a standards laboratory differential thermal voltage comparator (called a DTVC), [4] sensitive d-c null meter, and the average ac-dc converter under test. The magnitude of each harmonic of the source (up through the 9th) was measured. Its effect on the form factor of the voltage wave was calculated to be less than $10 \mathrm{ppm}$, assuming the worst possible phase angle with respect to the fundamental.

The standard was the rms responding DTVC unit, having the function of monitoring the sine-wave signal to be measured and matching it by null method to the d-c supply of the equivalent rms value. In turn the d-c supply was scaled down by a factor of $\sqrt{2} / \pi$, by the attenuator, and nulled against the $d$-c output of the average converter. The $d$-c difference is the error of 


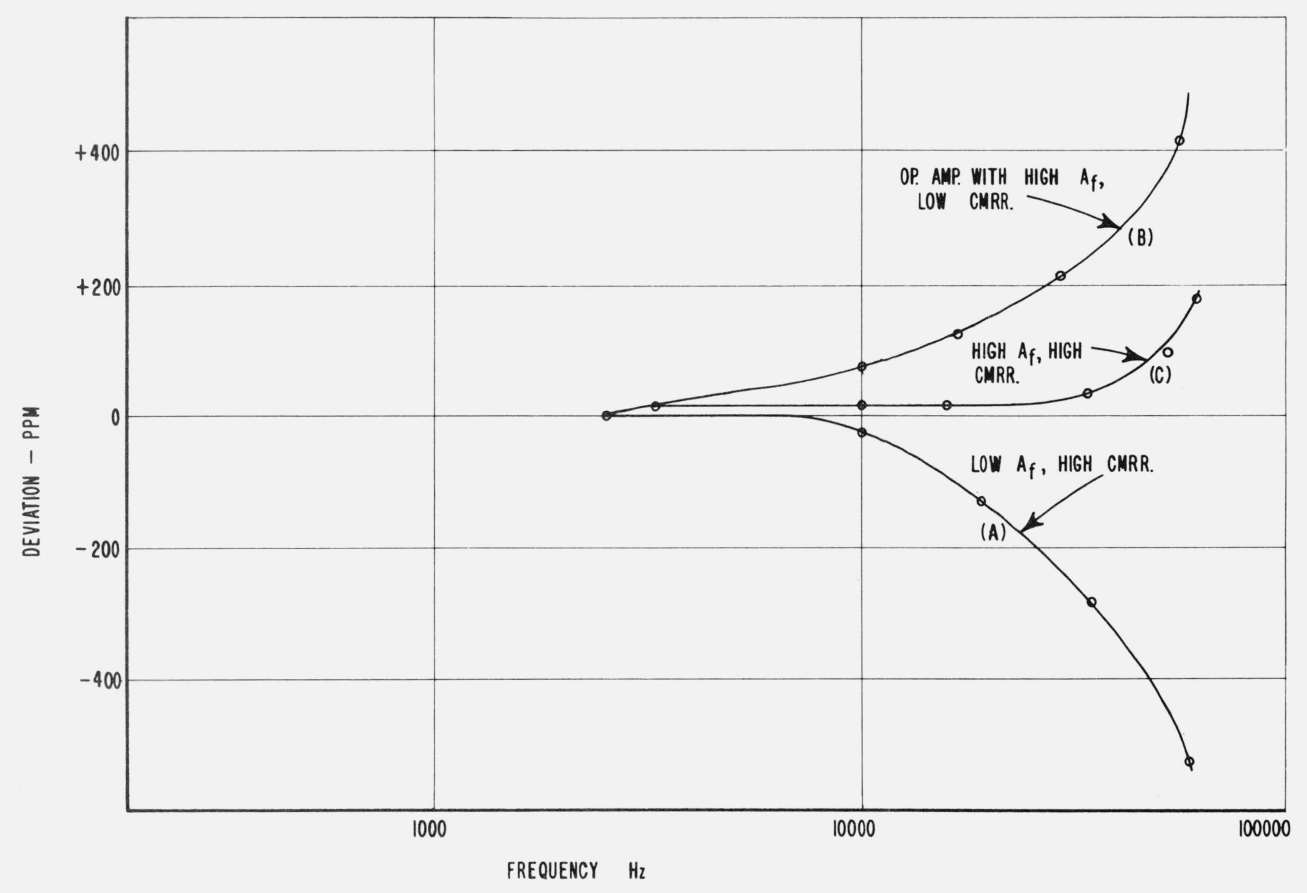

FigURE 4. Frequency response of figure 3.

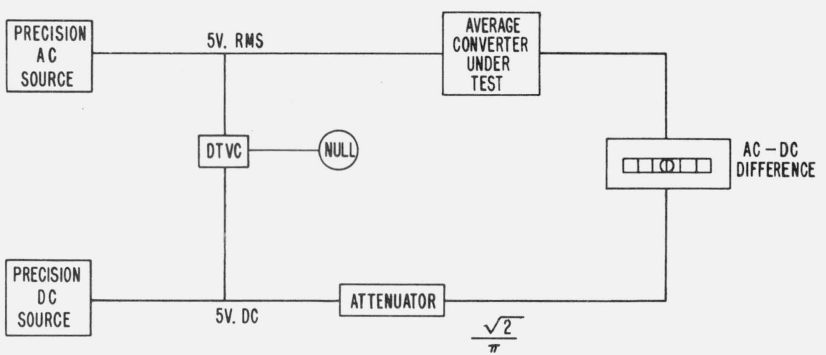

Figure 5. Testing system.

the device under test. Since the entire system has d-c response, scale factor inaccuracies at any position in the system could be checked by substituting the same d-c supply potential for the a-c signal (the average unit has identical input-output polarity correspondence). By using the same d-c supply as both signal and reference, its amplitude could be varied at will, allowing a check on dynamic range and on d-c offset. A $5 \mathrm{~V} \mathrm{rms} \mathrm{a-c} \mathrm{level} \mathrm{was} \mathrm{used} \mathrm{in} \mathrm{all} \mathrm{the} \mathrm{tests} \mathrm{in} \mathrm{order}$ to avoid approaching the usual $\pm 10 \mathrm{~V}$ operational amplifier output limit. Operating voltages below $5 \mathrm{~V}$ are not recommended because most of the error factors discussed would be increased.

The results of these tests in figure 6 show that the operational rectification and filtering combination could yield a d-c voltage whose amplitude can be related to the rms value of a-c input with an accuracy of better than $20 \mathrm{ppm}$ for frequencies out to $1000 \mathrm{~Hz}$. At higher frequencies the accuracy was degraded because this was a feed forward type of amplifier. Short term per- turbations of output were in the vicinity of $1 \mathrm{ppm}$, and possibly less.

These tests have demonstrated high accuracy performance at low and mid range audio frequencies. The next emphasis was on the design factors needed to extend the operating frequency range to $100000 \mathrm{~Hz}$ and to include a high input impedance stage ahead of the rectifier.

\subsection{Tests for Frequency Response}

This group of tests involved the latest differential FET operational amplifiers having wide-band, highvalue open-loop amplification figures. In addition, for reasons previously stated in the section on precision resistors, metal film resistance components were used in these tests. Since the question of measurement accuracy had been settled in the previously described group of tests, the test circuit arrangement was modified to demonstrate operational precision. Rather than employing the primary standard DTVC unit, a much simpler version of differential thermal comparator was used to monitor the output of the a-c signal power source for the purpose of maintaining constant amplitude. A peak ac-de voltage comparator, though sensitive to waveform distortion, could be used in place of an rms comparator since an ac source of low distortion is specified. An in-depth analysis of the effects of ac distortion on the rectified average value can be found in reference [3]. For these tests the stress was on frequency response, exact value of magnitudes being of secondary importance. The recorded values were the measured differences 


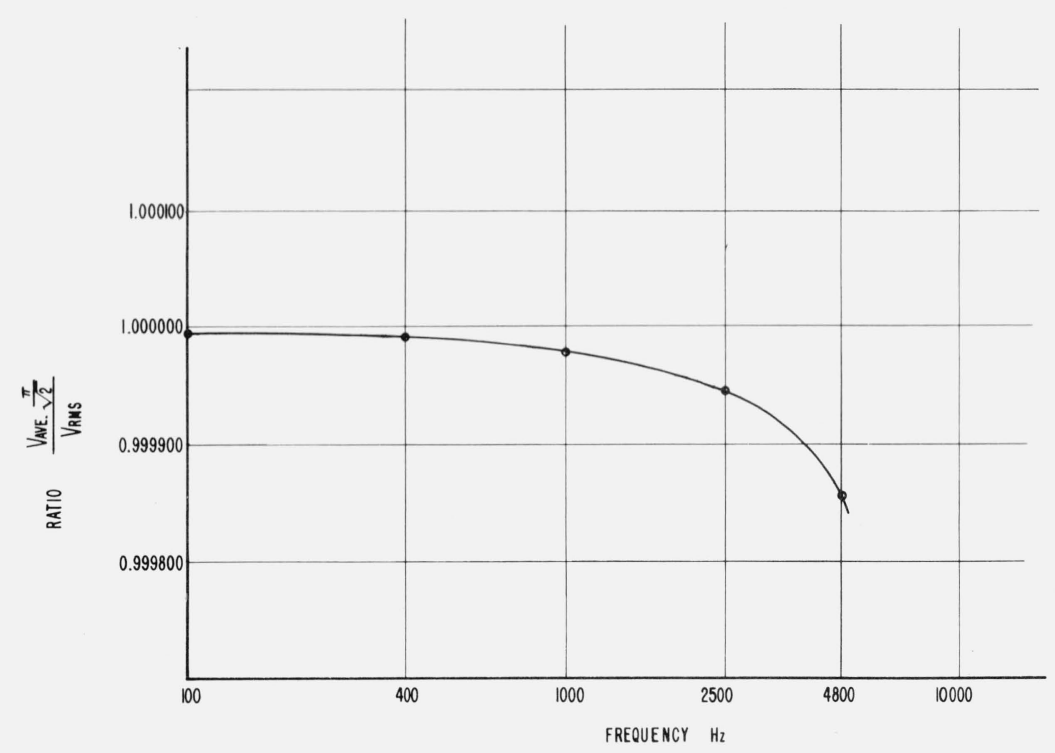

FigURE 6. Transfer accuracy of ac-dc converter (Chopper-stabilized amplifier).

between the average d-c output and the fixed d-c reference power supply, expressed in ppm and plotted versus frequency. Normalizing the error values at some low frequency starting point is a means of removing the error offset due to any imprecision of resistor ratio and $d$-c offset at the amplifier stages.

The crosses in figure 7 show the test values obtained with an operational rectifier-filter combination which had metal film resistors. ${ }^{2}$ Considering the number of instruments involved in the tests and their individual contribution to the end precision, the assignment of $\pm 150 \mathrm{ppm}$ for the wide-band ac-dc error of the average measuring instrument is conservative. At the same time of the tests shown in figure 7 , a unity-gain input isolation amplifier stage was added ahead of the operational rectifier state. In figure 7 , the circles show the results of three runs; again all the values taken are plotted. Comparison of the circles and crosses shows that very little was changed in the response characteristic with the addition of the isolating amplifier. The benefit realized is substantial; the impedance presented to the a-c source increased from $5 \mathrm{k} \Omega$ to $10 \mathrm{M} \Omega$. All frequency test points were within $200 \mathrm{ppm}$ of the normalized value.

\section{Some Wiring Suggestions}

Circuit wiring, especially in the operational rectifier circuit should be short for minimum lead inductance and arranged in a manner for minimum shunt capacitance. One of the most critical points in the connection from the selected polarity output of the rectifier to the input resistor of the filter stage. Distortion of the half-sine wave resulting from shunt capacitance at this point could affect the accuracy of the average value.

${ }^{2}$ Four tests were made at each frequency, but were not separately plotted when the results differed by only small amounts.
It is strongly suggested the electronic circuit be d-c isolated from chassis ground. Removing the input and output common terminal from ground helps in eliminating the noisy effects of circulating d-c ground currents. Both a-c and d-c isolation can be had by a guarded circuit which offers low electrical capacity from the common terminals to chassis. This form of isolation requires a box-within-a-box type construction plus a special power supply. Such regulated supplies with a multi-shielded transformer are available with 10000 megohm d-c insulation and $0.1 \mathrm{pF}$ line-power input to d-c output isolation.

\section{Initial D-C Offset Voltage Adjustments}

After a sufficient warm-up period (usually $1 \mathrm{hr}$ ) the voltage offset of the filter stage is trimmed for zero output voltage with its input connection from the input resistor momentarily tied to common. The operational rectifier stage offset can be likewise adjusted by shorting to common the input side of the input resistor and trimming for zero output voltage. For this stage the diodes must be temporarily short-circuited. Effectiveness of input-output transfer of the instrument can be observed by applying some d-c value from 1 to $10 \mathrm{~V}$ to the input and comparing it to the instrument output by means of a null meter; the diode rectifier polarity output must be correctly selected. The d-c inputoutput agreement should be within the ratio accuracy of the resistors at the rectifier and filter stages.

\section{Suggested Circuit}

Figure 8 is a schematic diagram of a line-up of operational stages for use as an average ac-dc converting instrument. The circuit is capable of serving either the need for accuracy or for operational precision. 


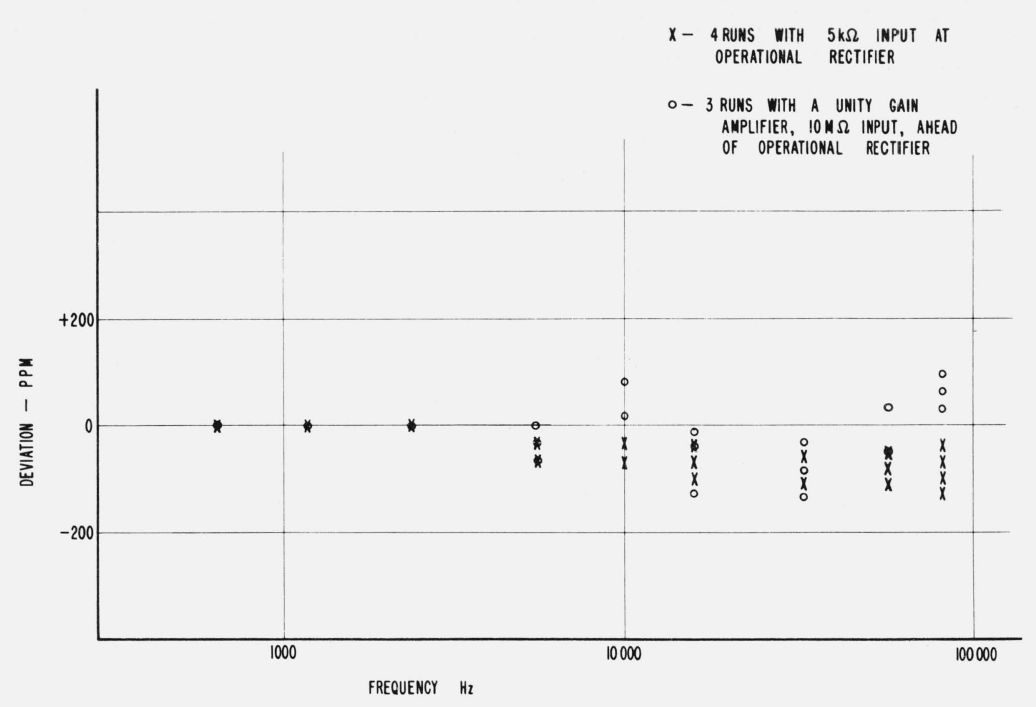

FIGURE 7. Frequency response of converter.

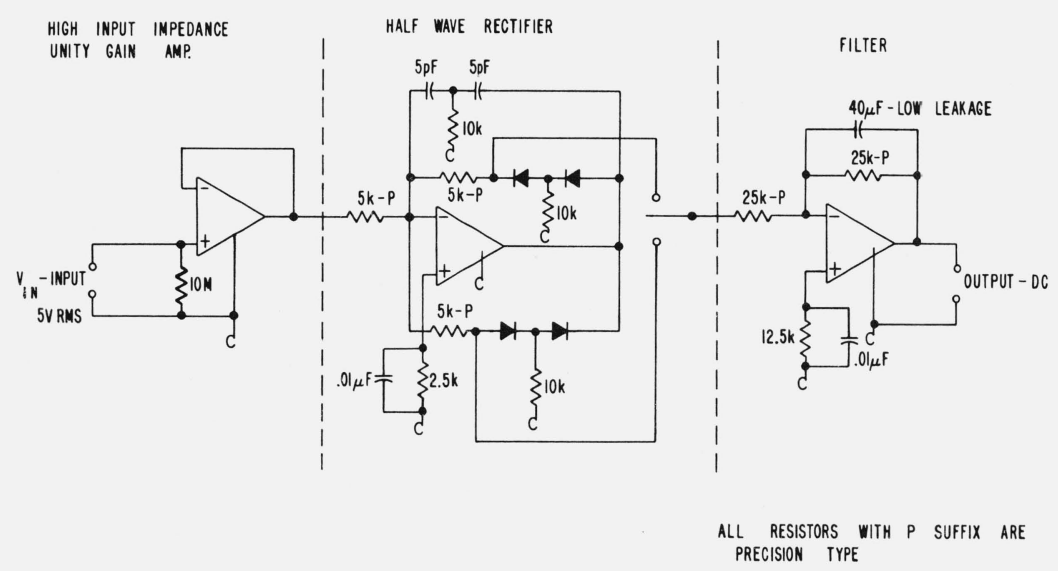

Figure 8. Schematic diagram.

If accuracy of $20 \mathrm{ppm}$ is desired for ac-dc transfer, wherein the measured values can be related on an rms basis, the following points are pertinent. The high input impedance operational first stage must be deleted and the input signal applied to the second stage - the operational rectifier. The reason for this precaution is that the available amplifiers for use as a noninverting stage have marginal open loop gain and CMRR figures for accuracy work below $100 \mathrm{ppm}$. As for the rectifier stage, the selected operational amplifier must have a very high open loop gain and a low d-c drift. An open loop gain of 200000 at $1 \mathrm{kHz}$ is needed if the contributed error is to be less than $10 \mathrm{ppm}$. The voltage drift should be in the area of $1 \mu \mathrm{V} /{ }^{\circ} \mathrm{C}$ and $10^{-10} \mathrm{~A} /{ }^{\circ} \mathrm{C}$ for the current drift. While differential FET type operational amplifiers come close to meeting these specifications, the accuracy results reported in this writing have been obtained with chopper stabilized devices. Because of their possible feed-forward design the $20 \mathrm{ppm}$ operating accuracy is limited to frequencies below $1 \mathrm{kHz}$ and the gain setting resistors at the rectifier stage are restricted to values of $1 \mathrm{k} \Omega$ or less. The latter components should have a ratio accuracy error of less than $5 \mathrm{ppm}$, available in oil filled, wire wound resistors. A signal level of $5 \mathrm{~V} \mathrm{rms}$ at the a-c source is suggested. The a-c signal power source should have a total harmonic distortion of less than 50 ppm and freedom from a d-c component, if the rms value is desired. The operational amplifier at the filter stage need only have the stated $\mathrm{d}$-c precision of the rectifier stage.

The three stages shown in figure 8 can offer an operating precision and flatness of amplitude response with frequency of better than $200 \mathrm{ppm}$ over the range from d-c to $100 \mathrm{KHz}$, as described previously under the section heading of Test Results. FET-type differ- 
ential operational amplifiers can be found to meet the requirements of each stage. If the sole interest is operational precision in the form of stability and repeatability, the principal concern in selecting the operational amplifiers is the $\mathrm{d}$-c drift characteristics. However, the open loop voltage gain has to be considered in order to maintain the desired transfer gain ratio. With a voltage drift value below $10 \mu \mathrm{V} /{ }^{\circ} \mathrm{C}$ and a current drift under $10^{-10} \mathrm{~A} /{ }^{\circ} \mathrm{C}$ the operating precision can be held to better than $100 \mathrm{ppm}$. For situations requiring accuracy of measurement or if flatness of amplitude response with frequency is important, the wide-band open loop voltage gain deserves additional attention. In the case of the noninverting first stage the CMRR figure is also important. If the input stage is to have a unity gain accuracy of $100 \mathrm{ppm}$ at $5 \mathrm{kHz}$, both the open loop gain and the CMRR at that frequency should be at least 20000 for the selected operational amplifier. For the rectifier stage the same values of 20000 is needed at $5 \mathrm{kHz}$ for $100 \mathrm{ppm}$ accuracy but the CMRR can be neglected. The filter stage as usual need only have the stated d-c operating characteristics. Again, in the case of rms measurements of accuracy the a-c signal source must have appropriate purity-no more than $200 \mathrm{ppm}$ total harmonic distortion for $100 \mathrm{ppm}$ transfer accuracy.

\section{References}

[1] Richman, P., Operational Rectifier, U.S. Patent No. 3311835 , March 28, 1967, assigned to Weston Instruments, Inc.

[2] Marzetta, L. A., A Peak AC-DC Voltage Comparator for Use in a Standards Laboratory, NBS Technical Note No. 280, issued January 17, 1966.

[3] Richman, P., Modern AC Voltage Calibration for Audio and Sub-Audio Frequencies, presented at 19th Annual ISA Conference, October 12-15, 1964, New York, Preprint No. 21, 4-2-64.

[4] Hermach, F. L., Griffin, J. E., and Williams, E. S., A System for Accurate Direct \& Alternating Voltage Measurements, IEEE Trans. on Instrumentation \& Measurement IM14 p215-224, 1965.

(Paper 73C3\&4-288) 